\title{
Synthesis of Multiarmed Thienylene-Tetrafluorophenylene Alternating Copolymers under Transition-Metal-Free Conditions
}

Takanobu Sanji,* Junko Kakinuma, and Tomokazu Iyoda

Iyoda Supra-Integrated Material Project, Exploratory Research for Advanced Technology (ERATO), Japan Science and Technology Agency (JST), and Tokyo Institute of Technology, 4259-S2-3

Nagatsuta, Midori-ku, Yokohama 226-8503, Japan

E-mail: sanji.t.aa@m.titech.ac.jp (TS)

\section{Experimental section (continued.)}

\section{Polymerization.}

Model polymerization with 2a. TBAF ( $1 \mathrm{M}$ THF solution, $15 \mu \mathrm{L}, 1.5 \times 10^{-2} \mathrm{mmol}, 5 \mathrm{~mol} \%$ ) was added to $2 \mathrm{a}(5.0 \mathrm{mg}, 0.016 \mathrm{mmol})$ in THF $(1 \mathrm{~mL})$ at room temperature. After stirring for $30 \mathrm{~min}$ at room temperature, a THF solution $(4 \mathrm{~mL})$ of $\mathbf{1}(150 \mathrm{mg}, 0.30 \mathrm{mmol})$ was added to the mixture at room temperature and then the mixture was stirred for $2 \mathrm{~h}$. After addition of ethanol $(0.2 \mathrm{~mL})$, the mixture was poured into methanol $(200 \mathrm{~mL})$. The precipitate was collected by centrifugation. A second cycle of dissolving-precipitation followed by freeze-drying gave polymer 3 as a yellow powder ( $87 \mathrm{mg}, 70 \%)$. A yellow powder: $M_{\mathrm{n}}=9400, \nexists=1.43$ (SEC relative to polystyrene standards); ${ }^{1} \mathrm{H} \mathrm{NMR}\left(\mathrm{CDCl}_{3}, 400\right.$ MHz) $\delta 7.54,6.90,6.72,4.04,1.44-1.28,0.96-0.89,0.05 ;{ }^{19} \mathrm{~F}\left\{{ }^{1} \mathrm{H}\right\} \mathrm{NMR}\left(\mathrm{CDCl}_{3}, 376 \mathrm{MHz}\right) \delta-139.11$. Polymerization with $2 \mathbf{b}$ (I-shaped). TBAF ( $1 \mathrm{M}$ THF solution, $11 \mu \mathrm{L}, 1.1 \times 10^{-2} \mathrm{mmol}$, $5 \mathrm{~mol} \%$ ) was added to $2 \mathbf{b}(5.9 \mathrm{mg}, 0.011 \mathrm{mmol})$ in THF $(1 \mathrm{~mL})$ at room temperature. After stirring for $30 \mathrm{~min}$ at room temperature, a THF solution ( $4 \mathrm{~mL})$ of $1(117 \mathrm{mg}, 0.23 \mathrm{mmol})$ was added to the mixture at room temperature and then the mixture was stirred for $2 \mathrm{~h}$. After addition of ethanol $(0.2 \mathrm{~mL})$, the mixture was poured into methanol $(200 \mathrm{~mL})$. The precipitate was collected by centrifugation. A second cycle of dissolving-precipitation followed by freeze-drying gave polymer 4 as a yellow powder (66 mg, 69\%). A yellow powder: $M_{\mathrm{n}}=7100, \nexists=1.71$ ( $\mathrm{SEC}$ relative to polystyrene standards); ${ }^{1} \mathrm{H} \mathrm{NMR}\left(\mathrm{CDCl}_{3}, 400\right.$ $\mathrm{MHz}) \delta 7.08,6.71,4.03,1.43-1.26,0.93-0.88,0.05 ;{ }^{19} \mathrm{~F}\left\{{ }^{1} \mathrm{H}\right\} \mathrm{NMR}\left(\mathrm{CDCl}_{3}, 376 \mathrm{MHz}\right) \delta-139.11$.

Polymerization with 2c (V-shaped). TBAF ( $1 \mathrm{M}$ THF solution, $20 \mu \mathrm{L}, 2 \times 10^{-2} \mathrm{mmol}, 5 \mathrm{~mol} \%$ ) was added to $2 \mathbf{c}(8.6 \mathrm{mg}, 0.021 \mathrm{mmol})$ in THF $(2 \mathrm{~mL})$ at room temperature. After stirring for $30 \mathrm{~min}$ at room temperature, a THF solution $(2 \mathrm{~mL})$ of $1(213 \mathrm{mg}, 0.420 \mathrm{mmol})$ was added to the mixture at room temperature and then the mixture was stirred for $2 \mathrm{~h}$. After addition of ethanol $(0.2 \mathrm{~mL})$, the mixture was poured into methanol $(200 \mathrm{~mL})$. The precipitate was collected by centrifugation. A second cycle of dissolving-precipitation followed by freeze-drying gave polymer 5 as a yellow powder (123 $\mathrm{mg}, 71 \%)$. A yellow powder: $M_{\mathrm{n}}=6000, \nexists=1.65$ (SEC relative to polystyrene standards); ${ }^{1} \mathrm{H} \mathrm{NMR}\left(\mathrm{CDCl}_{3}, 400\right.$ $\mathrm{MHz}) \delta 7.18,6.71,4.03,1.56-1.26,0.94-0.87,0.03 ;{ }^{19} \mathrm{~F}\left\{{ }^{1} \mathrm{H}\right\} \mathrm{NMR}\left(\mathrm{CDCl}_{3}, 376 \mathrm{MHz}\right) \delta-139.11$. 


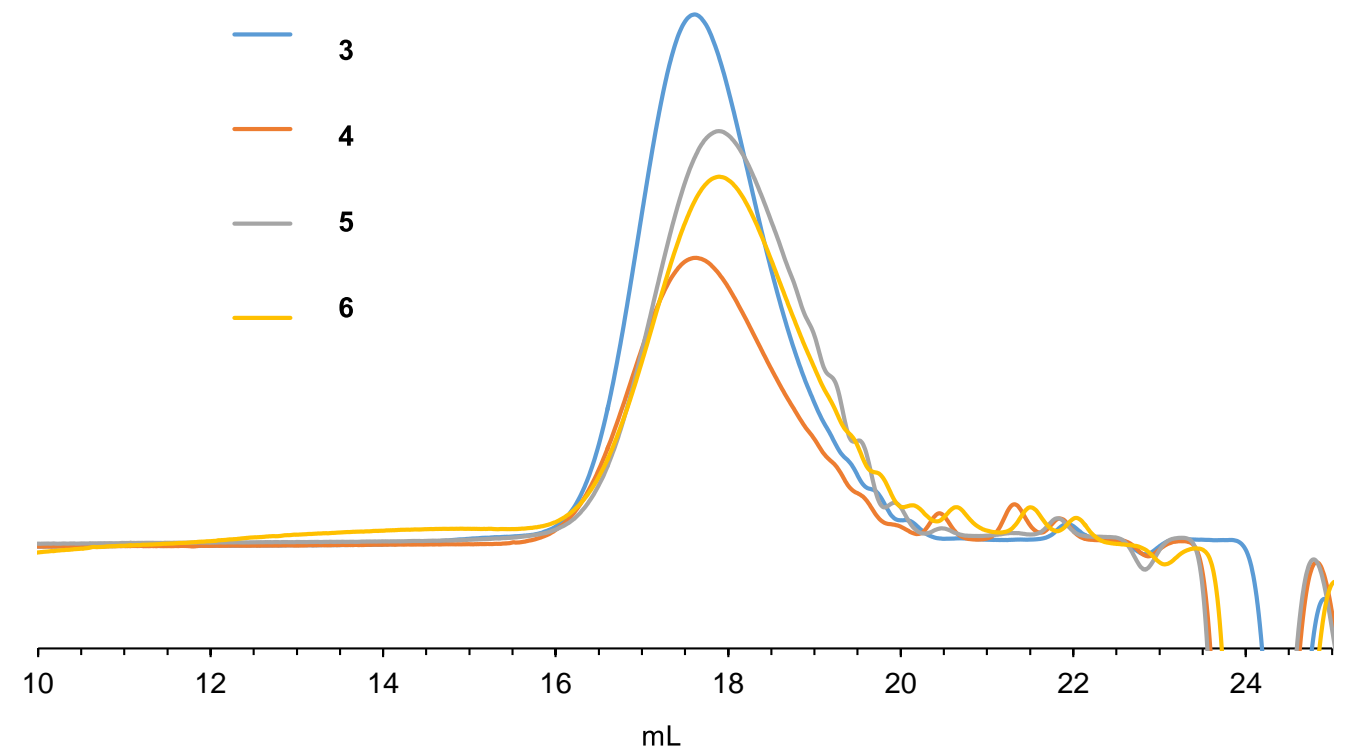

Figure S1. SEC curves (RI) of 3-6. 

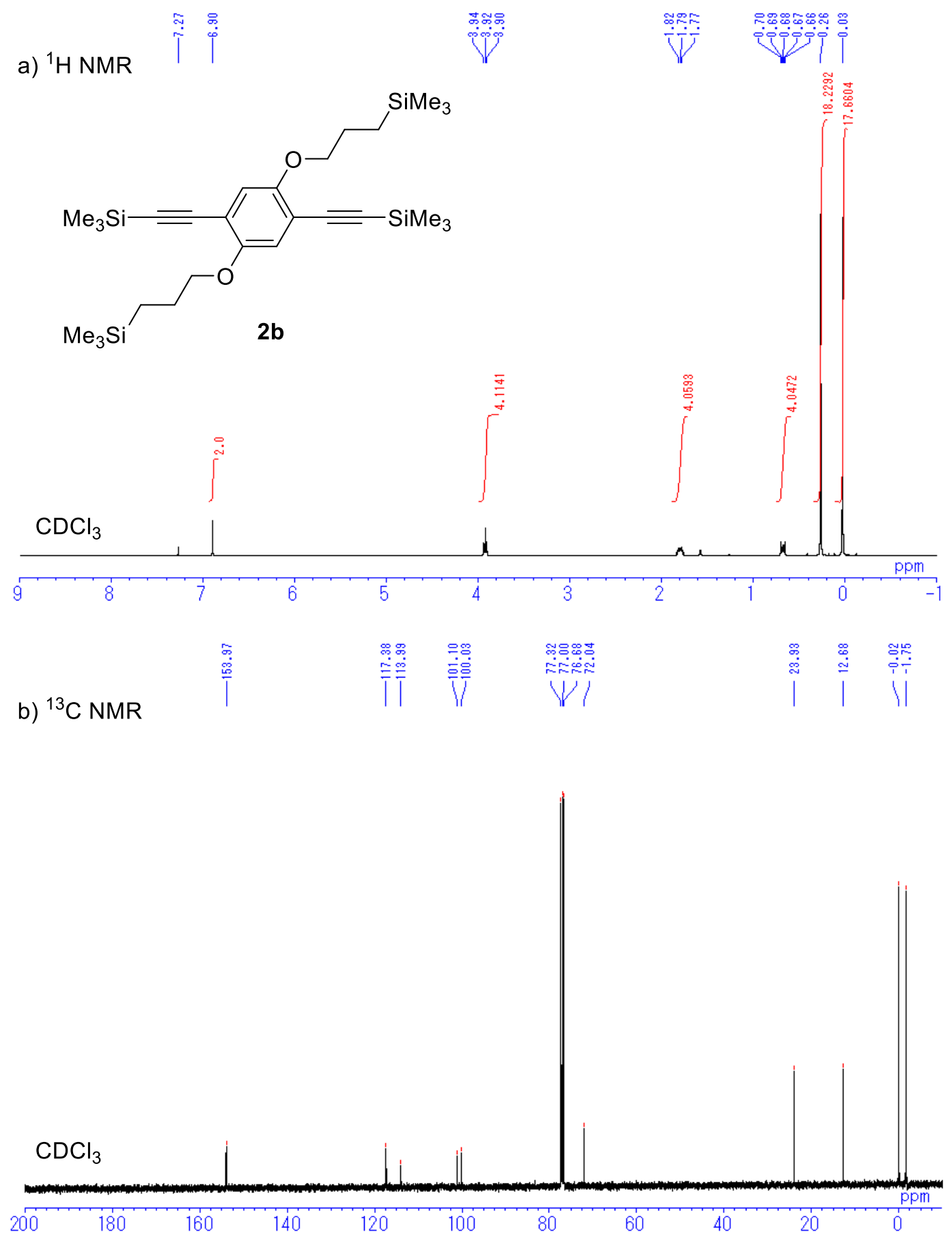
a) ${ }^{1} \mathrm{HNMR}$

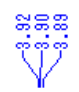

कृष्

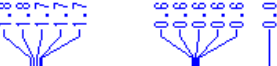<smiles>CSCCCOc1cc(Br)cc(Br)c1</smiles>

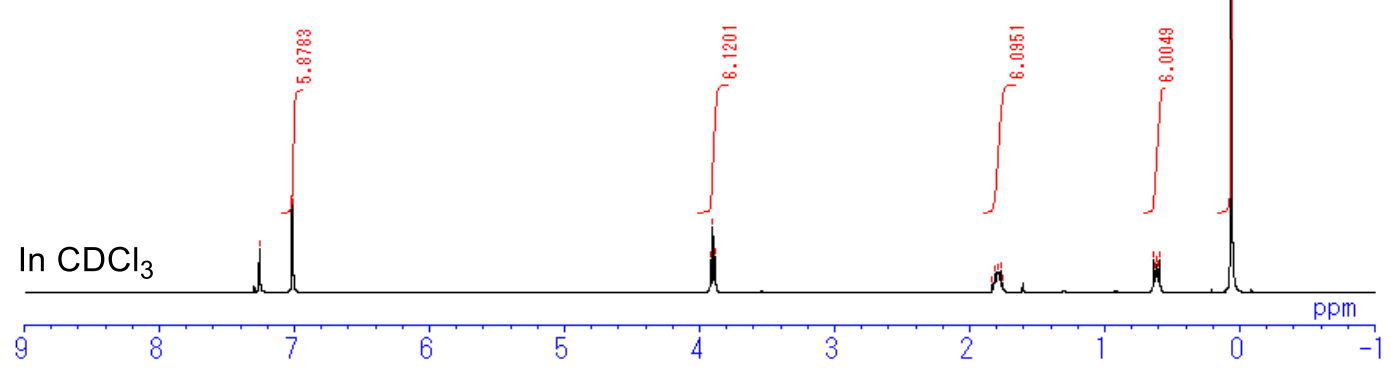

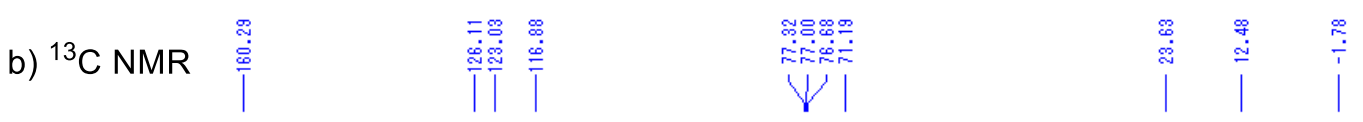

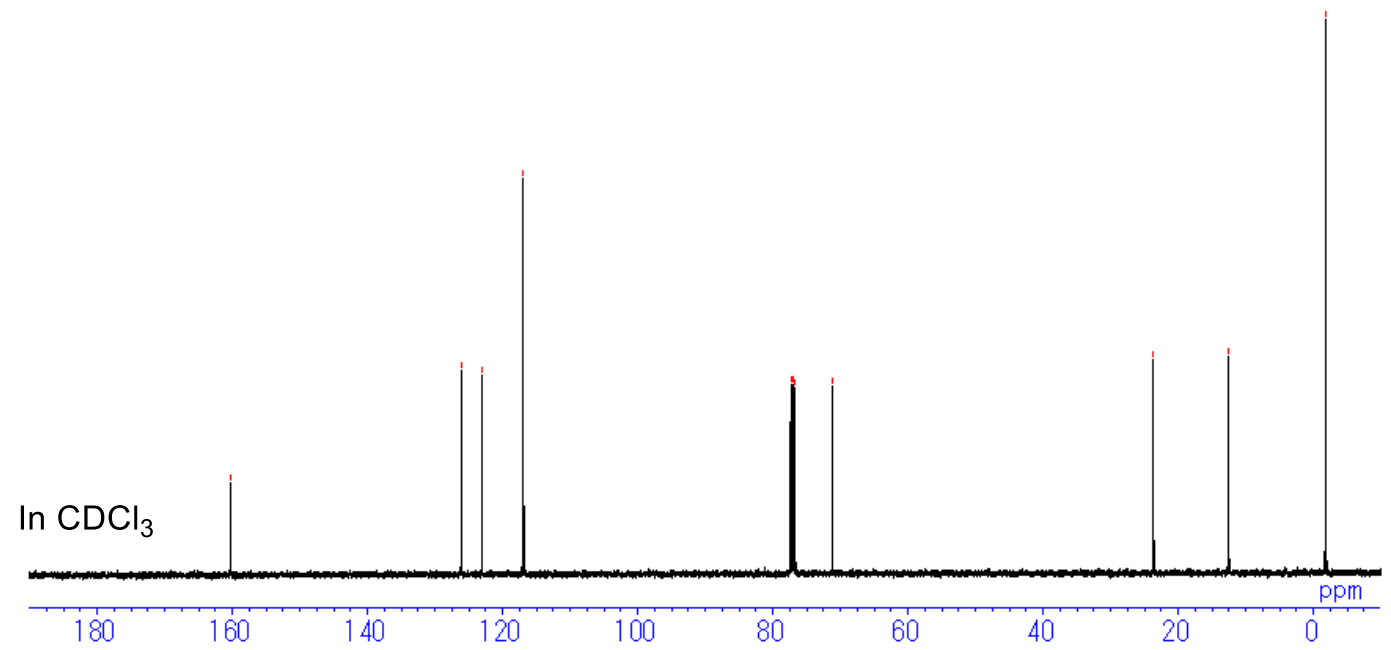


a) ${ }^{1} \mathrm{H}$ NMR
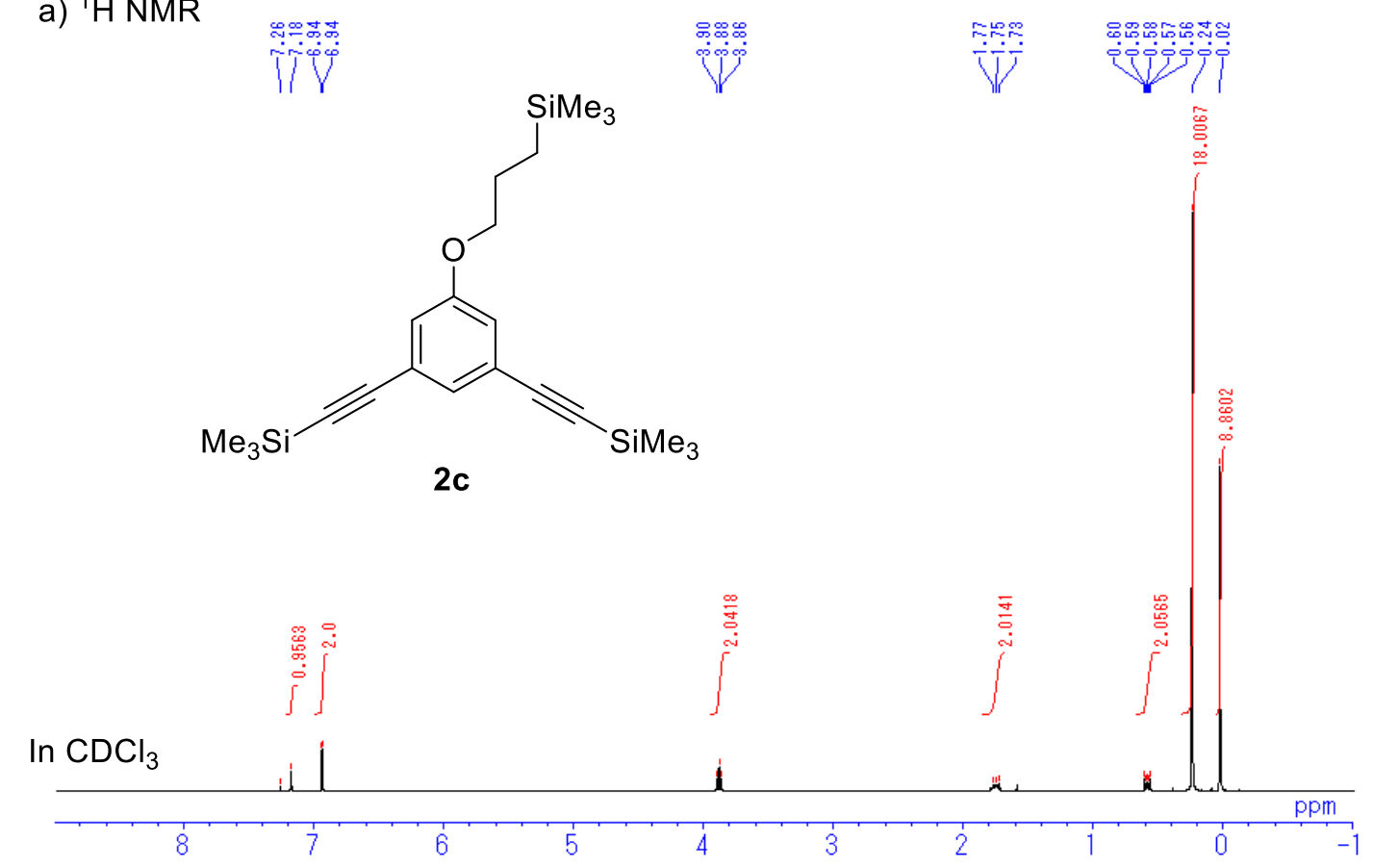

b) ${ }^{13} \mathrm{C} N M R$

\begin{tabular}{|c|c|c|c|c|c|}
\hline$\stackrel{\circ}{\circ}$ & $\Xi \pm$ & 启 & $\stackrel{\mathscr{O}}{ }$ & 尔 & యొర్రం\% \\
\hline$\stackrel{8}{\infty}$ & 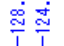 & $\stackrel{\infty}{=}$ & 过 & 市 & $\ddot{m} \dot{\theta} \dot{m}$ \\
\hline
\end{tabular}

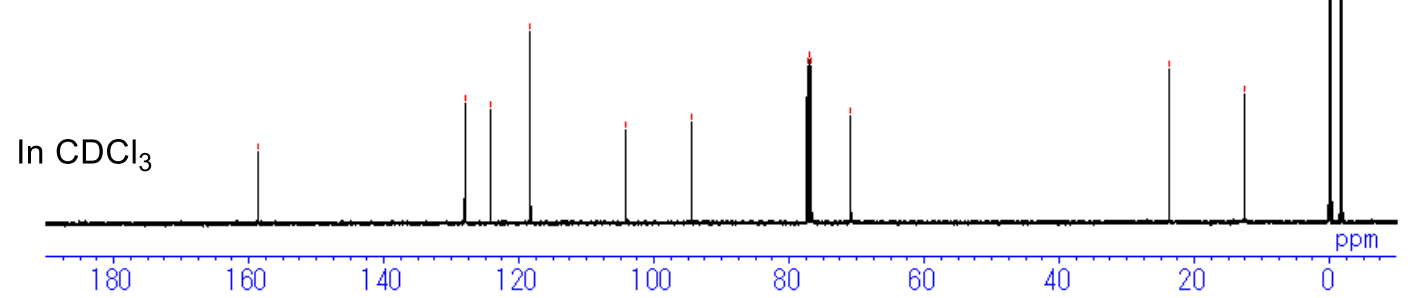




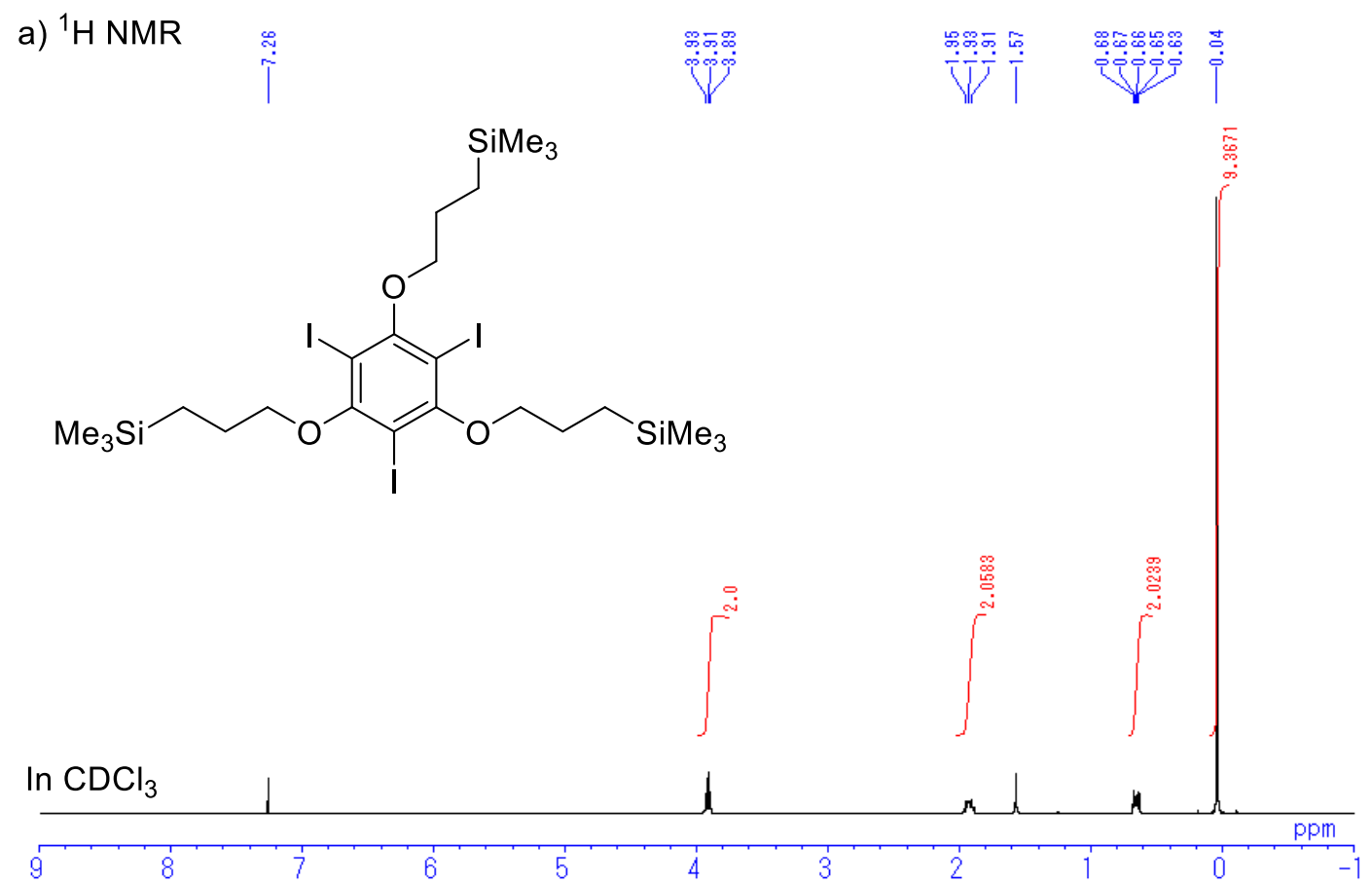
b) ${ }^{13} \mathrm{C}$ NMR
w.m.
IW

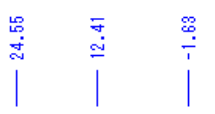

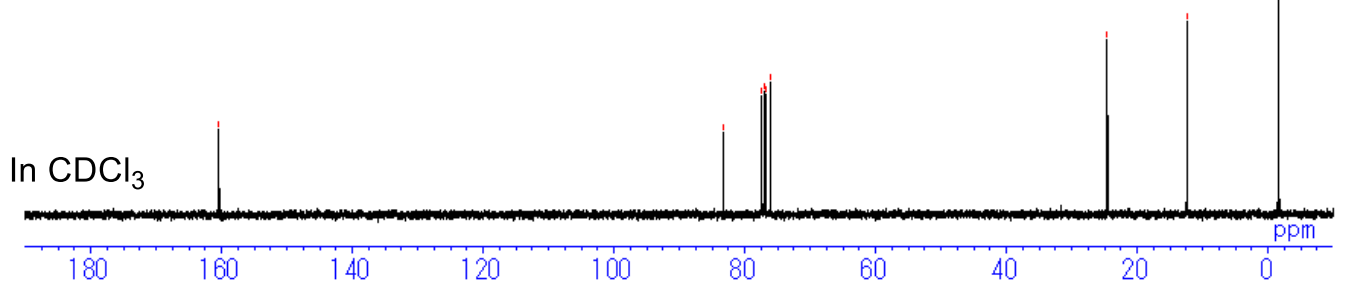


a) ${ }^{1} \mathrm{H}$ NMR

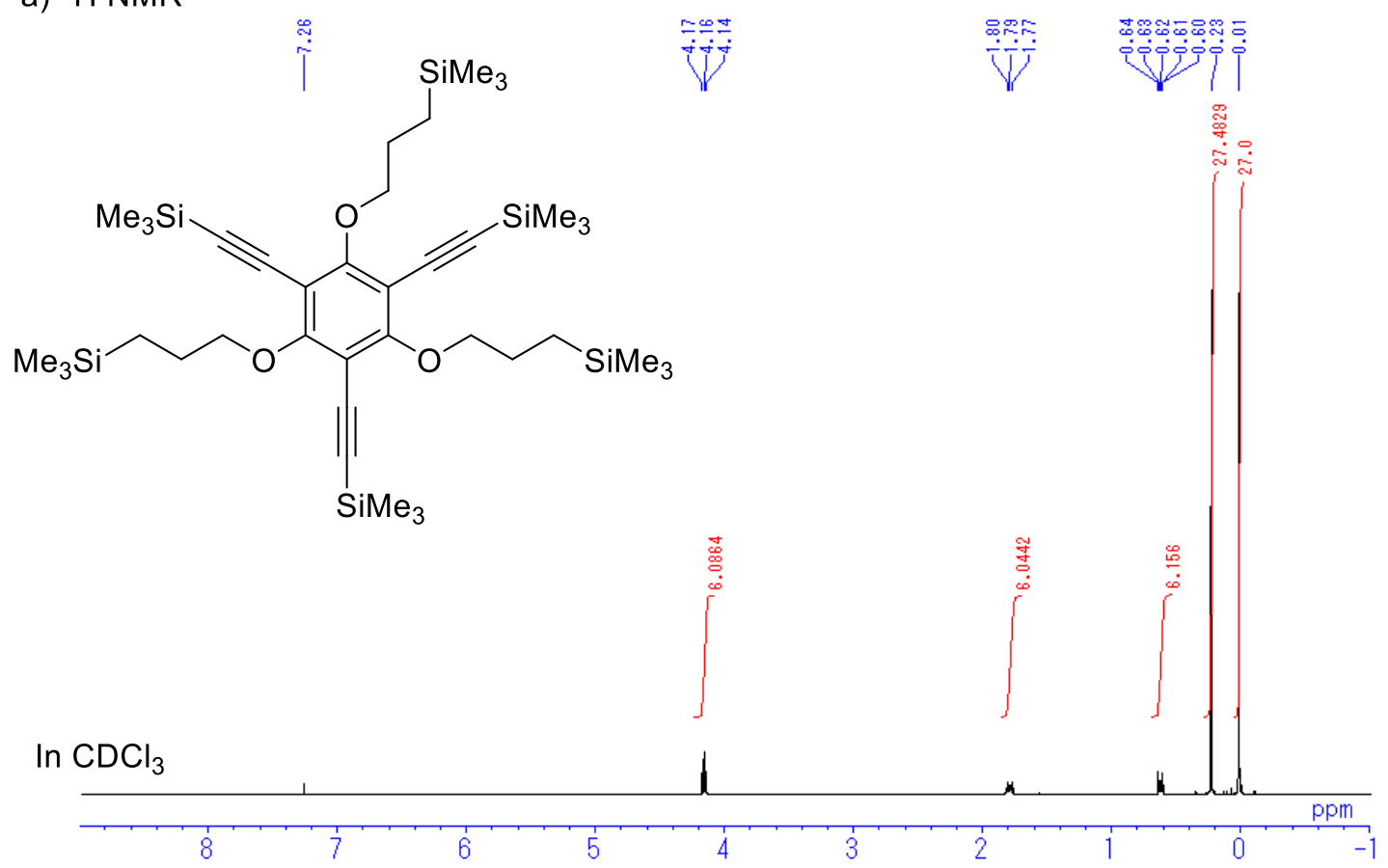

b) ${ }^{13} \mathrm{C}$ NMR

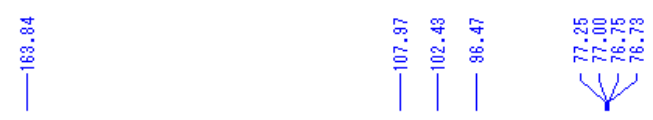

$\mid$

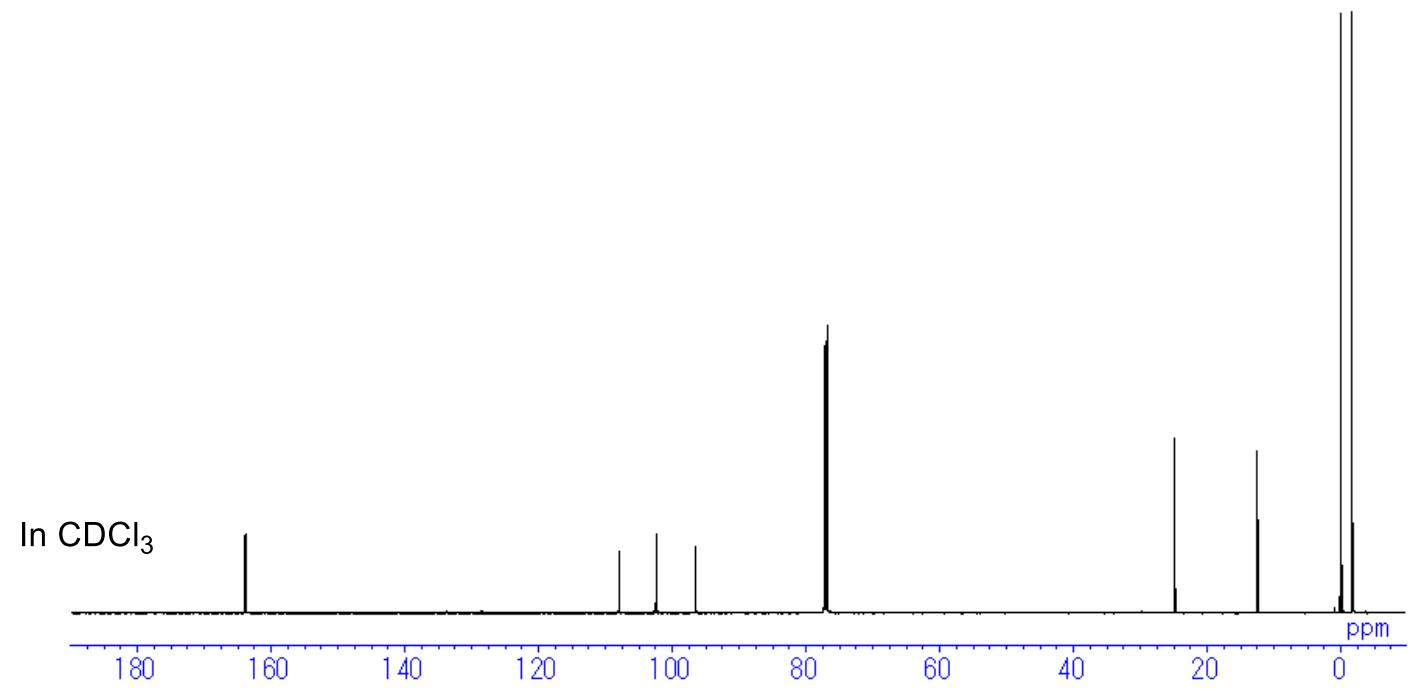

